\title{
Finite Element Evaluation of Effective Thermal Conductivity of Short Carbon Nano Tubes: a Comparative Study
}

\author{
Zia Javanbakht ${ }^{\mathrm{a}}$, Wayne Hall ${ }^{\mathrm{b}}$, Andreas Öchsner $^{\mathrm{c}}$ \\ School of Engineering, Griffith University, Gold Coast Campus, Southport 4222, Australia. \\ aZia.Javanbakht@gmail.com, bw.hall@griffith.edu.au ,,'Andreas.Oechsner@gmail.com
}

Keywords: Finite element method; Reinforced composites; Thermal analysis; Computational analysis.

\begin{abstract}
In the current study, two extreme cases are considered for the dispersion of carbon nanotubes (CNTs) in a polymeric matrix: randomly-oriented and randomly-aligned. The representative volume element (RVE) is used to represent the composite material consisting of epoxy resin matrix and CNTreinforcement. The finite element method acts as the computational tool to conduct the simulations and investigate the effective parameters, i.e., the influence of the aspect ratio and the orientation, on the thermal conductivity of the matrix. A Fortran subroutine was used for both generation and analysis of the models by means of the MSC Marc finite element package and a Python script was used for the sensitivity analysis. The results indicate that optimum performance of the CNTs in terms of thermal conductivity can be reached by orienting them along the temperature gradient whereas a random distribution improves the conductivity by a smaller magnitude.
\end{abstract}

\section{Introduction}

In the most general case, any combination of two or more materials with different properties and distinct boundaries is referred to as a composite material which provides the capability of adjusting the compliance of the whole material to some extent [1]. This enables the composite materials to be used in a variety of applications ranging from industrial and infrastructural forms to sports and musical instruments [2]. Basically, reinforcements are included in the composite material to compensate for the deficiencies of the matrix. Based on the type of the inclusion, fibrous, laminated, particulate, or any combination of these classes of composite materials can be produced [3].

Among all types of inclusion, fibers as 'pseudo one-dimensional fillers' [4] have been in practice since $4000 \mathrm{BC}$ but mostly on the meso-scale. Recent nano-technological advancements, e.g. the discovery of the CNTs in 1991 [5], have attracted a huge amount of research interest. Extremely high elastic modulus (greater than $1 \mathrm{TPa}$ ) and tensile strength (10-100 times that of the strongest steel) was reported for single walled carbon nanotubes with an average density of $1.33-1.44 \frac{\mathrm{g}}{\mathrm{cm}^{3}}$. This unusually high performance characteristics of the CNTs is remarkable when compared with other materials in the same range of density [6, 7]. From another perspective, a very high thermal conductivity $\left(6600 \frac{\mathrm{W}}{\mathrm{m} \cdot \mathrm{K}}\right)$ has also been reported for CNTs $[8,9,10,11]$. The generally high-standard diverse properties of the CNTs make them multi-functional reinforcing components for composites.

In terms of mediums for composite materials, average performance should be expected to justify the incorporation of any reinforcing agents such as the CNTs. Among the available materials as a matrix component, polymers demonstrate insufficient thermal properties which limits their applicability [9]. To overcome such issues, CNTs were introduced to fortify the somewhat mediocre properties of polymer matrices. Additionally, a direct approach to measure the properties of the CNTs is cumbersome and thus, measurements are generally done for the matrix-contained CNTs instead of the individual fiber [12]. The popularity of the computational methods in material characterisation of the composite can be partly attributed to such complications in the experimental methods.

The finite element method is used to solve a broad range of engineering boundary value problems among which are the multi-scale/multi-physics problems and the transfer phenomena of the continuum mechanics (mass transfer) and, more specific to the interest of this study, the thermodynamics 
(heat transfer) [13]. Several computational efforts were made to investigate the effective parameters on the thermal conductivity of the CNT-reinforced composites, e.g., mechanical loading [14], fiber aspect ratio $[15,16]$, fiber orientation $[17,18]$, fiber agglomeration $[19,20]$, and fiber volume fraction $[21$, 22].

One of the challenges in generating various fiber orientation, using the Monte-Carlo algorithm for instance (see $[23,24]$ ), is the requirement for a supporting programming language, such as Matlab, to generate the mesh (see [14] for instance). In the current study, not only an automatised approach is adopted but also the periodicity of the representative volume element (RVE) is considered to evaluate the effects of random and aligned dispersion of CNTs in a polymer-based composite.

\section{Methodology}

In the current study, the simulation of the heat transfer phenomenon within a fiber-reinforced composite medium was carried out by means of the finite element method [25, 26]. In order to customize the dispersion of the fibers, a commercial semi-open package, i.e., the MSC.Marc (version 2014.2) software, was used. The important feature of the package is its capability of incorporating personalized procedures via the Fortran programming language [27]. The subroutines were developed in Fortran which handled both the random- and aligned-dispersion of the fibers, automatically. However, a Python script was used to modify the mesh density, deal with the results and submit the job.

The periodicity condition was established for the $20 \times 20 \times 20 \mathrm{~nm}^{3}$ RVE in which the short fibers with a length of $10 \%$ of the RVE edge length, i.e., $2 \mathrm{~nm}$, were embedded. The effective cross sectional area of the short fibers were assumed to be $1.13 \mathrm{~nm}^{2}$ as a result of a wall thickness of $0.34 \mathrm{~nm}$, and an outer diameter of $1.4 \mathrm{~nm}$ [18]. The thermal conductivity of the individual components of the matrix is listed in Table 1.

Table 1: Thermal conductivity of the composite components

\begin{tabular}{ccc}
\hline Component & Material & $\begin{array}{c}\text { Conductivity } \\
\left(\frac{\mathrm{w}}{\mathrm{m} \cdot \mathrm{K}}\right)\end{array}$ \\
\hline Matrix & Epoxy resin & $0.214[18]$ \\
Fiber & Carbon nanotube & $2980[28]$ \\
\hline
\end{tabular}

The thermal analyses were carried out using a uniform mesh which consisted of two types of thermal elements: type 43 and type 36 . The former is a 8-node isoparametric hexahedral element which was used to represent the matrix whereas the latter is basically, the straight 2-node link/truss element representing the fibers. The prescribed temperature boundary conditions were applied to the top and bottom face nodes of the RVE to establish a temperature gradient within the matrix (see Fig. 1). The reaction heat flux of the top face nodes was accumulated and then, used to calculate the effective thermal conductivity of the composite by means of the Fourier's law [29]:

$$
\lambda=\frac{\dot{Q}}{A_{0}} \cdot \frac{\Delta z}{\Delta T}
$$

where $\lambda$ is the effective thermal conductivity of the composite, $\dot{Q}$ is the total reaction flux in the top boundary conditions, $A_{0}$ is the cross-sectional area perpendicular to direction of the flux, $\Delta z$ is the distance between the boundary faces, and $\Delta T$ is the prescribed temperature gradient.

Several sensitivity analyses were carried out by means of a Python script to obtain the optimum mesh density. Cubic hexahedral elements were used to discretise the RVE and thus, the uniform mesh density $\gamma$ can be calculated using the following equation [30]:

$$
\gamma=\frac{1}{a}
$$




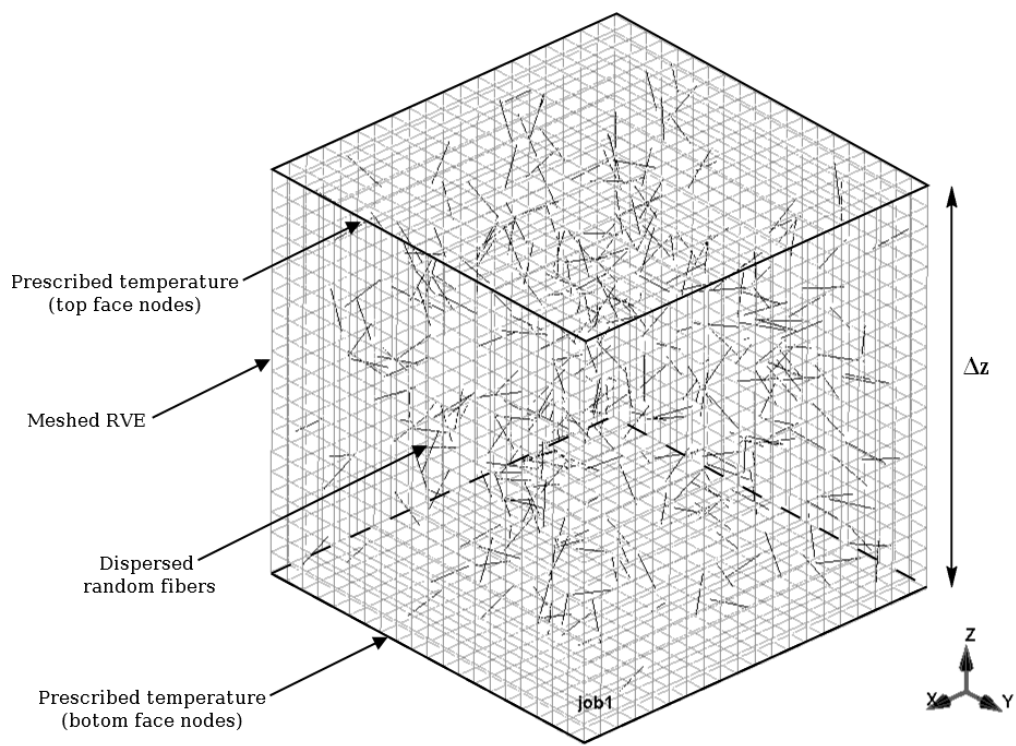

Fig. 1: Prescribed boundary conditions of the FE prototype (mesh density of 1)

where $a$ is the characteristic dimension of the element, i.e., the edge length of each cube. Several mesh densities, from 0.05 to 6 , were used to monitor the convergence of the effective thermal conductivity for the aligned fiber prototype.

In every job submission, the algorithm updated the random fiber distribution for each of the analysis cases. The length of each fiber is assumed and thus, for every fiber volume fraction, the number of fibers was calculate. In the case of the randomly oriented fibers, two set of coordinates were generated from which the end point coordinates were modified to set the correct length. If the end point was located outside of the RVE, the fiber was cut in its intersection point with the RVE and the remaining length was moved to the opposite face of the RVE. This ensured the periodicity condition of the RVE. A similar approach was adopted for the aligned fiber cases except that the direction of the fiber is already known. This procedure was repeated until reaching the required number of fibers (see Fig. 2). Note that the embedding mechanism of the software simplified the procedure, that is, no node matching or interface elements were used to incorporate the fibers into the matrix (see [31]).

Finally, it is worth mentioning that the nominal fiber volume fraction was used in the study, that is, the ratio of sum of the fiber volumes over the gross volume of the RVE. However, in order to be more accurate, the true fiber volume fraction is also calculated which is defined as the ratio of the fibers

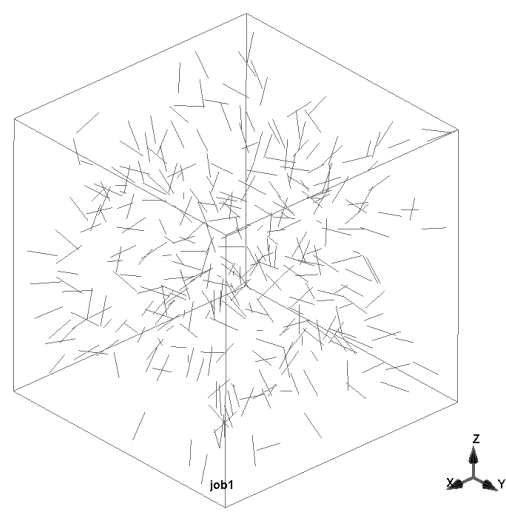

(a) Randomly dispersed fibers

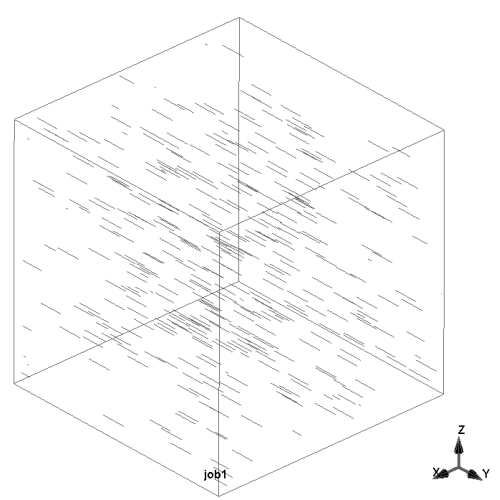

(b) Randomly dispersed aligned fibers along $Y$-axis

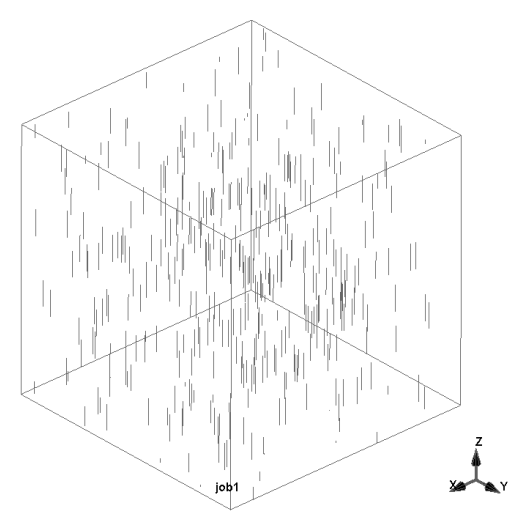

(c) Randomly dispersed aligned fibers along $Z$-axis

Fig. 2: RVE with randomly dispersed and aligned fibers 
over the net volume of the RVE. Namely, the net volume of the RVE was obtained by subtracting the total fiber volume from the gross RVE volume.

\section{Results and Discussion}

As a result of random fiber generation and meeting the periodicity condition, the number of total elements varies in each simulation. Therefore, the sensitivity of each specific fiber volume fraction was investigated individually. The target quantity was the effective thermal conductivity of the matrix. For each case, the relative error of the results was calculated with respect to those of the finest mesh, i.e., the results for the mesh density of 6 (see Table 2). The selection criterion of the best mesh was defined to be a maximum absolute relative error of $0.5 \%$. By increasing the number of fibers, that is, the nominal fiber volume fraction, the required mesh density was increased for maintaining the error within a certain threshold. Namely, the size of elements should be reduced by increasing the fiber volume fraction.

Table 2: Results of the sensitivity analysis

\begin{tabular}{ccc}
\hline $\begin{array}{c}\text { Nominal fiber } \\
\text { volume fraction } \\
(\%)\end{array}$ & $\begin{array}{c}\text { Uniform } \\
\text { mesh } \\
\text { density }\end{array}$ & $\begin{array}{c}\text { Relative } \\
\text { absolute error } \\
(\%)\end{array}$ \\
\hline 1 & 0.05 & 0.41 \\
2 & 1.60 & 0.49 \\
3 & 2.80 & 0.49 \\
4 & 3.40 & 0.48 \\
5 & 3.80 & 0.47 \\
6 & 4.20 & 0.46 \\
7 & 4.30 & 0.48 \\
8 & 4.60 & 0.47 \\
9 & 4.80 & 0.44 \\
10 & 4.80 & 0.48 \\
\hline
\end{tabular}

To achieve consistency between the prototypes of each simulation class, the finest required mesh was selected for all other cases, i.e., a mesh density of 4.8 corresponding to the nominal fiber volume fraction of $10 \%$. All the simulations, i.e., all the simulations for the two aligned fiber and one dispersed fiber cases, were conducted using the selected mesh density of 4.8. The periodicity of the RVE was also considered in the simulations. Each simulation was repeated 20 times per fiber volume fraction and the average value of the results were obtained (see Table 3 ).

The improvement in the average effective conductivity of the composite is noticeable — an impressive $25 \%$ improvement in average-for the aligned fibers along the $Z$-axis whereas in the $Y$-direction no improvement is observed, i.e., the conductivity of the reinforced matrix remains the same as that of the pure matrix. It is worth noticing that the improvements are almost independent of the fiber volume fraction. On the other hand, the randomly-oriented fibers demonstrate slight improvements in the equivalent thermal conductivity - a maximum of $4.06 \%$ in the highest fiber volume fraction. Obviously, the amount of improvement seems to depend on the magnitude of the fiber volume fraction. In post-processing of the results, the true fiber volume fraction deviates from the nominal fiber volume fraction as the volume of the fibers increases which may be influential in high volume fractions (see Fig. 3). 
Table 3: Simulation results of effective thermal conductivity for aligned and dispersed fibers for various fiber volume fractions

\begin{tabular}{|c|c|c|c|c|c|c|}
\hline \multirow[b]{2}{*}{$\begin{array}{l}\text { Nominal } \\
\text { fiber volume } \\
\text { fraction }(\%)\end{array}$} & \multicolumn{3}{|c|}{ Average effective conductivity $\left(\frac{\mathrm{W}}{\mathrm{m} \cdot \mathrm{K}}\right)$} & \multicolumn{3}{|c|}{ Improvement (\%) } \\
\hline & $\begin{array}{l}\text { Aligned } \\
\text { along } \\
Z \text {-axis }\end{array}$ & $\begin{array}{l}\text { Aligned } \\
\text { along } \\
Y \text {-axis }\end{array}$ & Dispersed & $\begin{array}{c}\text { Aligned } \\
\text { along } \\
Z \text {-axis }\end{array}$ & $\begin{array}{c}\text { Aligned } \\
\text { along } \\
Y \text {-axis }\end{array}$ & Dispersed \\
\hline 1 & 0.21663 & 0.21400 & 0.21490 & 26.04 & 0.0 & 0.42 \\
\hline 2 & 0.21915 & 0.21400 & 0.21566 & 25.24 & 0.0 & 0.78 \\
\hline 3 & 0.22163 & 0.21400 & 0.21648 & 24.67 & 0.0 & 1.16 \\
\hline 4 & 0.22484 & 0.21400 & 0.21740 & 26.02 & 0.0 & 1.59 \\
\hline 5 & 0.22738 & 0.21400 & 0.21827 & 25.42 & 0.0 & 2.00 \\
\hline 6 & 0.23050 & 0.21400 & 0.21922 & 25.85 & 0.0 & 2.44 \\
\hline 7 & 0.23243 & 0.21400 & 0.22011 & 24.49 & 0.0 & 2.85 \\
\hline 8 & 0.23575 & 0.21400 & 0.22099 & 25.01 & 0.0 & 3.27 \\
\hline 9 & 0.23851 & 0.21400 & 0.22182 & 24.78 & 0.0 & 3.66 \\
\hline 10 & 0.24048 & 0.21400 & 0.22269 & 23.83 & 0.0 & 4.06 \\
\hline
\end{tabular}

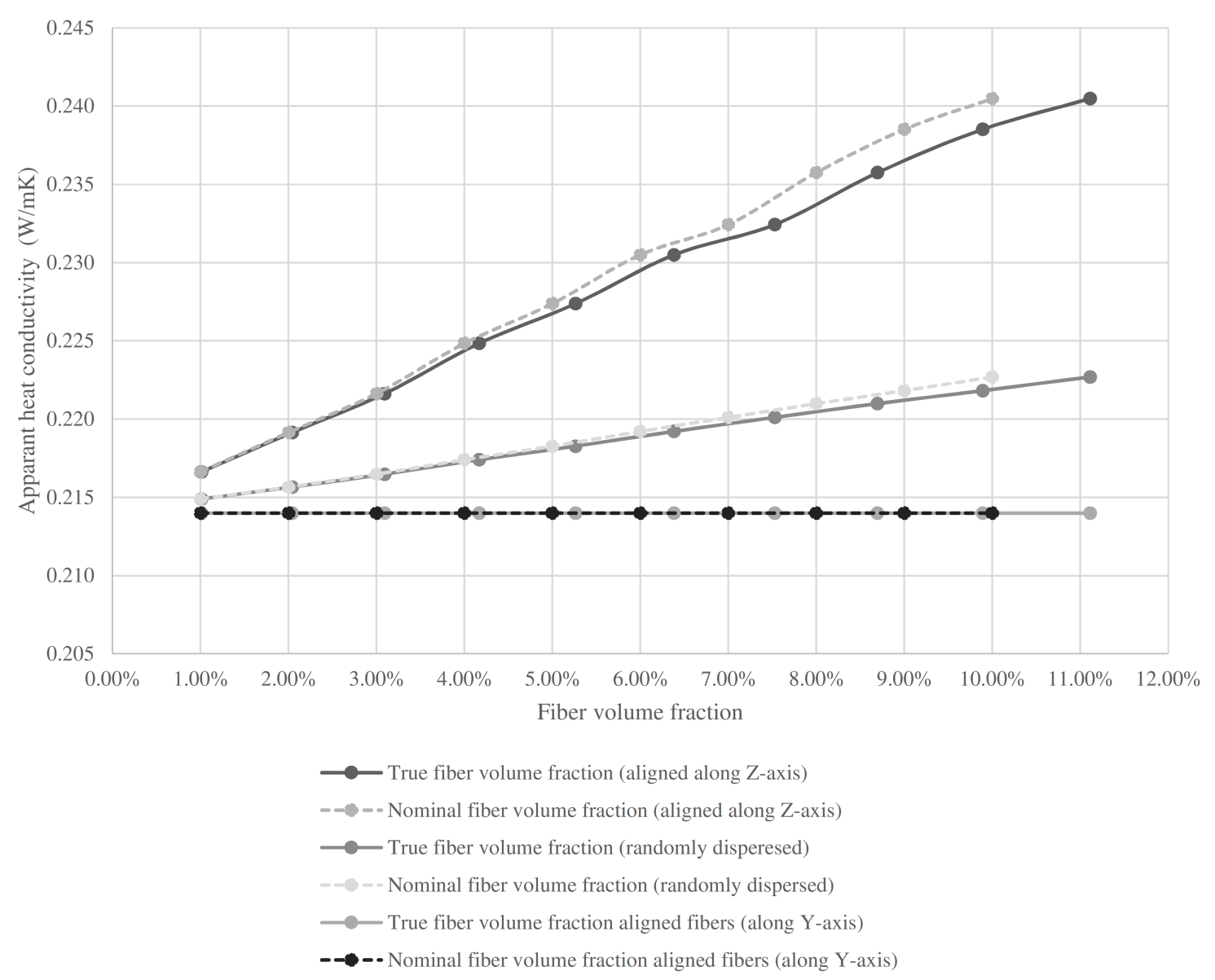

Fig. 3: Results of the simulations based on 20 repetitions per fiber volume fraction

\section{Conclusions}

In this study, three cases of random fiber dispersion was considered: aligned parallel to the boundary conditions (along the $Y$-axis), aligned perpendicular to the boundary conditions (along the $Z$-axis), and randomly dispersed fibers. The generation of short fibers was done by means of Fortran subroutines 
and a Python script. The simulations were repeated 20 times for each case and the average results investigated. The periodicity condition was also considered to improve the results. The higher fiber volume fraction made the FE model more sensitive to the mesh density in terms of the effective thermal conductivity. In addition, adding short fibers to the matrix increased the thermal conductivity of the composite in both cases of random dispersion and aligned along the $Z$-axis - for a maximum relative value of $26.04 \%$ and $4.06 \%$, respectively. In contrast, no improvement was observed for the aligned case along the $Y$-axis. It can be concluded that the optimised direction of the short fibers is along the temperature gradient while random distribution provides improvement to some extent. Finally, the deviation of the true fiber volume fraction values from the nominal ones is highly pronounced in high volume fractions which may be of importance in critical calculations.

\section{References}

[1] V. V. Vasiliev and E. V. Morozov. Advanced mechanics of composite materials. 2nd edn. Amsterdam and London: Elsevier, 2007.

[2] J. R. Vinson and R. L. Sierakowski. The behavior of structures composed of composite materials. 2nd ed. Vol. 105. Solid mechanics and its applications. Dordrecht and London: Kluwer Academic Publishers, 2002.

[3] R. M. Jones. Mechanics of composite materials. 2nd ed. Philadelphia, Pa. and London: Taylor \& Francis, 1999.

[4] J. N. Coleman et al. Small but strong: A review of the mechanical properties of carbon nanotubepolymer composites. Carbon 44 (2006), pp. 1624-1652.

[5] S. Iijima. Helical microtubules of graphitic carbon. Nature 354 (1991), pp. 56-58.

[6] E. T. Thostenson, Z. Ren, and T.-W. Chou. Advances in the science and technology of carbon nanotubes and their composites: A review. Compos. Sci. Technol. 61 (2001), pp. 1899-1912.

[7] E. T. Thostenson, C. Li, and T.-W. Chou. Nanocomposites in context. Compos. Sci. Technol. 65 (2005), pp. 491-516.

[8] S. Berber, Y.-K. Kwon, and D. Tománek. Unusually High Thermal Conductivity of Carbon Nanotubes. Phys. Rev. Lett. 84 (2000). May, pp. 4613-4616.

[9] Z. Han and A. Fina. Thermal conductivity of carbon nanotubes and their polymer nanocomposites: A review. Prog. Polym. Sci. 36 (2011), pp. 914-944.

[10] N. Behabtu et al. Strong, light, multifunctional fibers of carbon nanotubes with ultrahigh conductivity. Science 339 (2013), pp. 182-186.

[11] R. H. Baughman, A. A. Zakhidov, and W. A. de Heer. Carbon nanotubes-the route toward applications. Science 297 (2002), pp. 787-792.

[12] R. S. Ruoff and D. C. Lorents. Mechanical and thermal properties of carbon nanotubes. Carbon 33 (1995), pp. 925-930.

[13] H. Chen et al. Thermal conductivity of polymer-based composites: Fundamentals and applications. Prog. Polym. Sci. 59 (2016), pp. 41-85.

[14] M. Mahdavi et al. Effective thermal and mechanical properties of short carbon fiber/natural rubber composites as a function of mechanical loading. Appl. Therm. Eng. 117 (2017), pp. 816.

[15] H. R. Lusti and A. A. Gusev. Finite element predictions for the thermoelastic properties of nanotube reinforced polymers. Modelling Simul. Mater. Sci. Eng. 12 (2004), S107-S119.

[16] I. E. Afrooz and A. Öchsner. Effect of the Carbon Nanotube Distribution on the Thermal Conductivity of Composite Materials. J. Heat Transfer 137 (2015), p. 034501.

[17] N. Khani, M. Yildiz, and B. Koc. Elastic properties of coiled carbon nanotube reinforced nanocomposite: A finite element study. Mater. Des. 109 (2016), pp. 123-132. 
[18] R. Makvandi and A. Öchsner. On a Finite Element Approach to Predict the Thermal Conductivity of Carbon Fiber Reinforced Composite Materials. Defect. Diffus. Forum 354 (2014), pp. 215-225.

[19] S. R. Bakshi, R. R. Patel, and A. Agarwal. Thermal conductivity of carbon nanotube reinforced aluminum composites: A multi-scale study using object oriented finite element method. Comput. Mater. Sci. 50 (2010), pp. 419-428.

[20] H. S. Hedia et al. Effect of agglomeration and dispersion on the elastic properties of polymer nanocomposites: A Monte Carlo finite element analysis. MP 58 (2016), pp. 269-279.

[21] K. Alasvand Zarasvand and H. Golestanian. Determination of nonlinear behavior of multiwalled carbon nanotube reinforced polymer: Experimental, numerical, and micromechanical. Mater. Des. 109 (2016), pp. 314-323.

[22] X. L. Chen and Y. J. Liu. Square representative volume elements for evaluating the effective material properties of carbon nanotube-based composites. Comput. Mater. Sci. 29 (2004), pp. 111.

[23] I. V. Belova et al. The Lattice Monte Carlo Method for Solving Phenomenological Mass and Thermal Diffusion Problems. Defect. Diffus. Forum 279 (2008), pp. 13-22.

[24] I. V. Belova et al. The lattice Monte Carlo method for solving phenomenological mass and heat transport problems. DDC 4 (2007), pp. 15-1.

[25] A. Öchsner and M. Merkel. One-Dimensional Finite Elements: An Introduction to the FE Method. Berlin and Heidelberg: Springer, 2013.

[26] A. Öchsner. Computational statics and dynamics: An introduction based on the finite element method. Singapore: Springer, 2016.

[27] Z. Javanbakht and A. Öchsner. Advanced finite element simulation with MSC Marc: Application of user subroutines. Cham: Springer, 2017.

[28] T. Fiedler, E. Solórzano, and A. Öchsner. Numerical and experimental analysis of the thermal conductivity of metallic hollow sphere structures. Mater. Lett. 62 (2008), pp. 1204-1207.

[29] T. Fiedler et al. A refined finite element analysis on the thermal conductivity of perforated hollow sphere structures. Comput. Mater. Sci. 47 (2009), pp. 314-319.

[30] Z. Javanbakht, W. Hall, and A. Öchsner. Automatized Estimation of the Effective Thermal Conductivity of Carbon Fiber Reinforced Composite Materials. Defect. Diffus. Forum 370 (2016), pp. 177-183.

[31] MSC Software Corporation. Marc 2015: Theory and User Information. Vol. A. Newport Beach, California: MSC Software Corporation, 2015. 